\title{
The 5-factor model of psychological response to COVID-19: its correlation with anxiety and depression
}

\author{
Mohammad A. Aboul-ata ${ }^{1}$ (D) Faten T. Qonsua ${ }^{1}$ \\ Accepted: 31 March 2021 / Published online: 14 April 2021 \\ (C) The Author(s), under exclusive licence to Springer Science+Business Media, LLC, part of Springer Nature 2021
}

\begin{abstract}
The present study aimed to investigate the psychological response to COVID-19 on a community Egyptian sample and the correlation thereof with anxiety and depression. Further, we aimed to explore the differences in the psychological response and psychopathology in terms of demographics. We developed a COVID-19 Psychological Response Questionnaire (CPRQ). Further, we estimated the factor structure and internal consistency of CPRQ. Patient Health Questionnaire-9 (PHQ-9) was utilized to assess Major Depressive Disorder (MDD). Generalized Anxiety Disorder-7 (GAD-7) was utilized to assess Generalized Anxiety Disorder (GAD). The sample comprised 7015 participants whose ages ranged between 18 and 70 years. The present study results yielded a 5-factor model of psychological response to COVID-19 (the factors are Anxiousness, Social Support, Avoiding/Cleaning, Depressivity, and Medical Concern). In terms of reliability, the internal consistency of the above factors was acceptable except for Depressivity. There were significant positive correlations between two factors (Anxiousness and Depressivity) and both MDD and GAD. In terms of demographic characteristics, females had the highest values on Anxiousness, Avoiding/Cleaning, Depressivity, MDD, and GAD. Generally, the 5-factor model reflects the psychological response to COVID-19 during the period of the outbreak. Further, this model reflects several concepts regarding models and theories of health-related behaviors.
\end{abstract}

Keywords COVID-19 $\cdot$ Psychological response model $\cdot$ EFA $\cdot$ Generalized anxiety disorder $\cdot$ Major depressive disorder

\section{Introduction}

\section{Theories and Models of Health-Related Behaviors}

Understanding health-related behaviors is a basic aim of health psychology (Ogden, 2004, p. 4). Several models have been developed to explore and interpret the psychological response toward health events. In this context, Engel (1977) has proposed the biopsychosocial model to interpret the response to medical events. This model explains the interaction between biological, psychological, and social factors that shapes the response to specific health event. Moos and Schaefer (1984) have developed the "Crisis Theory".

Mohammad A. Aboul-ata

mohammad_aboulata@art.kfs.edu.eg

Faten T. Qonsua

faten.amer@art.kfs.edu.eg

1 Department of Psychology, Kafrelsheikh University, Kafr el-Sheikh, Egypt
They have proposed this theory to deal with a significant health event as a crisis event. According to such theory, the psychological response may vary in terms of dimension between healthy (adjustment) and unhealthy (maladjustment) behaviors.

Regarding health beliefs as the core of psychological response to health events, many researchers have investigated the health-related behaviors through several theories and models. One of such theories, the attribution theory, which investigated individuals' attributions of responsibility against their health conditions (Kelley, 1967). Further, the unrealistic optimism model explained individuals' perceptions of susceptibility to infections (Weinstein, 1983, 1984). In addition, the stages of change model provides a dynamic model for behavior changing depending on the individuals' evaluations of the benefits and costs of their behaviors (Prochaska \& DiClemente, 1982).

Other structured models have been raised as an integrated perspective for interpreting the psychological response toward health events. One of those is the health belief model, this model provides some core beliefs about illness (e.g., susceptibility, severity, costs, benefits, and cues to action) as 
predictors for healthy and unhealthy behaviors (Becker et al., 1977). Moreover, the protection motivation theory has been considered as an update for the health belief model. In such model, the researchers have added new factors that predict the health-related behaviors (Rogers, 1975, 1983). Further, the theory of planned behavior proposes that a combination of several beliefs configure the plans of action toward the targeted health-related behavior (Ajzen, 1985).

\section{Current Health Event (COVID-19 Outbreak)}

It is important to consider the psychological influence of COVID-19 besides the physical symptoms (Zhou et al., 2020). In China, the outbreak of COVID-19 resulted in psychological distress and triggered public fear. Such stress may result from uncertainty about the extent of this crisis and problematic consequences thereof (Bao et al., 2020; Yao et al., 2020; Zhang et al., 2020). Spreading false or inaccurate information about COVID-19 through social media may exaggerate the psychological pressure in facing such pandemic (Pennycook et al., 2020). In this regard, Qian et al. (2020) have estimated the public perception and psychological responses to COVID-19 among a sample of 510 participants from Wuhan and 501 from Shanghai. That study indicated that false and unreliable information on COVID-19 was the main cause behind such a high level of anxiety. In addition, Kwok et al. (2020) have investigated the risk-perception and community psychological response to COVID-19. Results reflected participants' high risk-perception towards COVID19. Furthermore, among Chinese citizens who frequently exposed to social media about COVID-19, the study showed a high prevalence of depression, anxiety, and comorbidity depression (Gao et al., 2020).

\section{Psychopathology and COVID-19 Outbreak}

Anxiety, depression, and other psychological consequences of the COVID-19 outbreak have affected all segments of society (e.g., patients, health professionals, and public) (Yang et al., 2020). In Italy, this pandemic has led to serious implications with notable psychological distress (Lazzerini \& Putoto, 2020). Also, such an outbreak caused a high prevalence of Posttraumatic Stress Disorder (PTSD) reaching 7\% among 285 residents in Wuhan and surrounding cities. Further, such a study indicated that PSTD was more prevalent in females rather than males (Liu et al., 2020).

\section{Demographics and Psychopathology during COVID-19 Outbreak}

A study on the undergraduates of Changzhi Medical College (China) showed that $0.9 \%$ of respondents suffered severe anxiety. Moreover, results revealed that some factors (e.g. living in urban areas, family income stability, and living with parents) are considered protective measures against anxiety. Besides, quarantine procedures have correlated positively with anxiety (Cao et al., 2020). Huang et al. (2020) have investigated the psychological impact of COVID-19 among nurses and nursing students and found that women suffer severe anxiety as compared to men. Moreover, participants who live in urban areas showed a higher prevalence of anxiety than those who live in rural areas. The study of Qiu et al. (2020) estimated the prevalence of psychological distress such as panic, anxiety, post-traumatic, and depression disorders in a large sample included 52,730 participants from 36 Chinese provinces and surrounding places. The results showed that females have higher levels of psychological distress than males. Furthermore, the 18-30 years-old group showed higher rates of stress rather than other ages. Also, the higher educated participants revealed a higher tendency to have more stress. In another study, results showed that women have more knowledge and positive practices in facing COVID-19 (Zhong et al., 2020).

\section{Mental Health in Egypt during COVID-19 Pandemic}

In Egypt, many researchers have investigated the impact of COVID-19 pandemic on mental health among various segments of society. Regarding the medical community, the findings reported that Egyptian physicians suffered high levels of anxiety and depression during this crisis (Abdelghani et al., 2020; Arafa et al., 2021; Khalaf et al., 2020). In terms of prevalence, about $50 \%$ of physicians had severe psychological problems during this crisis (Sehsah et al., 2021). Further, approximately $36 \%$ of Egyptian physicians showed a high degree of burnout syndrome (Abdelhafiz et al., 2020b). In a study investigating a sample of Egyptian Healthcare Workers $(\mathrm{HCW})$, findings showed their increasing risk perception and fear of infection due to the lack of personal protective equipment, as well as the likelihood to infect their family members (Abdel Wahed et al., 2020). Moreover, a high proportion of Egyptian HCW showed symptoms of depression (77.2\%), anxiety (76.4\%), stress (80.9\%), and insomnia (67.7\%), majority of which were females and those working in fever hospitals (Elkholy et al., 2020). Such symptoms were observed more frequent in females and younger healthcare professionals (Youssef et al., 2020). Further, in Zagazig (Egypt), during COVID-19 pandemic, nurses working at fever hospitals suffered higher stress levels than those working at general hospitals. Such finding was due to the perceived fear of COVID-19 infection and consequences thereof (Said \& El-Shafei, 2020).

Regarding the psychiatric morbidity of Egyptian youth, Alamrawy et al. (2021) found that most of participants showed symptoms of depression (80.5\%), anxiety (74.0\%), and insomnia (73.8\%) during the pandemic. As for severe 
symptoms, Arafa et al. (2020) have found a high prevalence of depression (22.5\%), anxiety (22.9\%), stress (15\%), and sleep deprivation $(23.1 \%)$ among the public in Egypt during COVID-19 outbreak. Among adult Egyptians, El-Zoghby et al. (2020) have reported that COVID-19 outbreak had adverse psychological consequences such as stress related to work, financial status and home. On the other hand, the study discovered some positive aspects such as mutual social support with family members and friends. Findings of another study investigated Egyptian adults revealed that quarantine procedures adversely affected mental wellbeing and caused stress among $70 \%$ of participants (Saleh, 2020). Further, Ghazawy et al. (2020) have investigated the psychiatric disorders during COVID-19 pandemic on a sample of Egyptian university students. Similarly, they have found that most of the participants suffered symptoms of depression, anxiety and stress. Further, they have reported that lack of psychological support and history of chronic disease may increase such symptoms especially in females. Another study has explored the perception and knowledge of the Egyptian public towards the COVID-19 outbreak. The findings showed that majority of participants recognized the risk of infection and committed by the protective measures (Abdelhafiz et al., 2020a).

\section{The Study Aims}

According to the Information and Decision Support Center (IDSC) of the Egyptian Cabinet (as of May 26, 2020) (see https://www.care.gov.eg/EgyptCare/Index. aspx), there were 17,967 confirmed cases of Covid-19, 783 deaths, and 4900 recovered in Egypt. The prevalence of COVID-19 in Egypt has increased dramatically in a few months (from March to May 2020). Consequently, such figures increased psychological distress (e.g., fear of infection and suffering quarantine procedures). Therefore, investigating psychological responses resulting from the COVID-19 outbreak is a valuable aim to reach under such pandemic circumstances, through detecting the factors that may lie behind the response to the COVID-19 pandemic. The present study aims to:

1- Conceptualize how participants respond to this crisis, as a psychological response to health event in a valid model and compare such findings with other relevant health models.

2- Estimate the correlation between the psychological response model and both depression and anxiety.

3- Investigate the differences in the psychological response model, depression, and anxiety among sample subgroups (using demographics)
The current study consists of three folds. The first is to construct a new questionnaire to assess the psychological response to the COVID-19 outbreak, as well as to provide a valid model that may contribute to exploring psychological response for such a critical health event. The second is to investigate the psychological response toward the COVID19 outbreak and the correlation thereof with depression and anxiety. The third is to estimate the differences among study sample subgroups, which were categorized based upon demographic characteristics (e.g., gender and age).

\section{Method}

\section{Participants and Procedures}

Using a cluster random sampling technique (selected through online classrooms at our University), we selected 10 colleges from Kafrelsheikh University (as the only within the city, this University represents the whole Governorate). We selected colleges representing major disciplines at such University. From each college, we selected four grades and from each grade, we randomly selected 5 males and 5 females that represent the geographical boundaries of the Governorate. This procedure revealed 400 participants, 9 of which have been excluded due to non-response, and we eliminated one participant to keep sample homogeneity in terms of gender. Therefore, the sample consisted of 390 participants (195 Males and 195 Females, representing the geographical boundaries of our Governorate). Their ages ranged between 18 and 24 years $(M=19.91$ and $S D=1.47)$. Their socioeconomic status was $0.8 \%$ very low, $4.6 \%$ low, $69.7 \%$ moderate, $23.1 \%$ high, and $1.8 \%$ very high (47.2\% from urban areas and $52.8 \%$ from rural areas). We asked each student to send the CPRQ's link to his/her social network (e.g., friends, parents, parents' friends, grandparents, grandparents' friends, friends of friends, etc.) via available internet apps (e.g., WhatsApp and Facebook) under specific instructions as possible as they could follow. Such instructions were as follows:

1- Please, send this link to those who may concern to participate in the study as a volunteer.

2- Try your best to keep gender equality (i.e., if you sent the link to a male volunteer, try to send it to a female with similar, if not exact, demographic characteristics).

3- Try as much as possible to send the link to volunteers close to where you live.

4- Ask other volunteers to send the link to other participants under the same instructions.

We administered the CPRQ (25 items), the Patient Health Questionnaire-9 (PHQ-9), and the Generalized Anxiety Disorder-7 (GAD-7) by sending a Google Forms link to 
institutional emails (University Email) of such selected students. Such online administration has been conducted between May 29 (14,229 confirmed cases and 879 deaths) and June 18, 2020 (50,437 confirmed cases and 1938 deaths) (see: https://www.worldometers.info/coronavirus/country/egypt/). Receiving responses from participants depended on the rate thereof per day. When the rate lowered (e.g., between 1 to 2 responses per day for a week) we deactivated the link. Finally, the study sample comprised 7015 participants, whose demographic characteristics are detailed in Table 1. Finally, as of January 29, 2021 (at the time of finishing this study), there have been 164,871 confirmed cases and 9217 deaths (Confirmed cases 1645/million - deaths 94/million) (see: https://www.worldometers.info/coronavirus/\#countries).

\section{Measurements}

\section{Constructing the "COVID-19 Psychological Response Questionnaire" (CPRQ)}

We aimed to construct CPRQ to estimate "how people react cognitively (thinking) and behaviorally (acting) during this crisis?". We can conclude the item selection procedure for CPRQ as follow:

Table 1 Sample Descriptive

\begin{tabular}{lllr}
\hline$n=7015$ & & $\mathrm{~N}$ & $\%$ \\
\hline Gender & Male & 3402 & 48.50 \\
& Female & 3613 & 51.50 \\
Residence & Rural & 3459 & 49.3 \\
Ages & Urban & 3556 & 50.7 \\
& $18-21$ & 3738 & 53.3 \\
& $22-30$ & 2292 & 32.7 \\
& $31-40$ & 556 & 7.9 \\
SES & $41-50$ & 264 & 3.8 \\
& $51-60$ & 121 & 1.7 \\
& 60+ & 44 & 0.6 \\
& Very low & 140 & 2.0 \\
& Low & 568 & 8.1 \\
Education & Moderate & 4599 & 65.6 \\
& High & 1344 & 19.2 \\
& Very high & 364 & 5.2 \\
& Read \& write & 260 & 3.7 \\
& High School & 2044 & 29.1 \\
& University & 4424 & 63.1 \\
& Master & 219 & 3.1 \\
& PhD & 68 & 1.0 \\
\hline
\end{tabular}

Note: SES = Socioeconomic Status
1- On March 20, 2020 (5 days after quarantine started, there have been 285 confirmend cases and 8 deaths) (see https://www.worldometers.info/coronavirus/country/ egypt/), via Google Classroom (an online platform), we informed students of the Psychology Department, Kafrelshiekh University on voluntary participation in a study that aims to investigate actions and thoughts thereof about COVID-19 and related consequences.

2- We selected 100 participants using a cluster random sampling technique online (from undergraduate and postgraduate students in the Department of Psychology). We randomly selected 10 males and 10 females from each grade (4th Grade), yielding 80 participants. Further, we selected 5 males and 5 females from Master students and the same from $\mathrm{Ph}$.D. students that yielded 20 participants. Finally, the sample consisted of 50 males and 50 females, whose ages ranged between 18 and 36 years old $(m=22.73$ $S D=4.50)$.

3- We reviewed and concluded their responses in 25 items (the English Version, See Table 2)

4- We chose the 5-point Likert type scale as a response key next to every item $(1=$ Never to $5=$ Always, more details in Table 2)

5- We created the scale (self-report form) using Google Forms and generated the link.

\section{Patient Health Questionnaire-9 (PHQ-9)}

The PHQ-9 is a self-report scale for adults consists of nine items that reflect nine symptoms of Major Depressive Disorder based on DSM-IV diagnostic criteria (American Psychiatric Association, 2000). The PHQ-9 is utilized in assessing the severity of the symptoms of depression. Sawaya et al. (2016) have translated, culturally adapted, and evaluated the psychometric properties of the PHQ-9 in Arabic culture. They found good reliability with Cronbach's Alpha of 0.88 . and high item-total correlation (ranged between 0.62 and 0.77). Further, their results revealed a two-factor solution (mood and somatic symptoms). Furthermore, AlHadi et al. (2017) evaluated the internal consistency of the Arabic Version of PHQ-9 and found that Cronbach's Alpha was 0.857. Finally, the Arabic Version of PHQ-9 was found to be psychometrically sufficient to be utilized in this study for screening depressive symptoms.

\section{Generalized Anxiety Disorder-7 (GAD-7)}

The GAD-7 is a self-report scale for adults consists of seven items that reflect seven symptoms of Generalized 
Table 2 The translated version (into English) of the CPRQ

How do you think/behave during COVID-19 outbreak?

Please, choose the suitable answer from the given items. Note that there no right or wrong answers, just express yourself. Your identity will remain confidential and this is just a voluntary survey.

By answering this survey, you acknowledge that you accept to participate.

Thank you in advance

1- How dangerous is COVID-19 to human life?

( $1=$ Not dangerous at all, $5=$ Highly dangerous $)$

2- Risk of infection is

$(1=$ Very low, $5=$ Extremely high $)$

3- Can you avoid infection?

( $1=$ I cannot, $5=$ Easily)

4- Does your family help in avoiding infection?

$(1=$ Never, $5=$ Completely $)$

5 - Do you help your family to avoid infection?

( $1=$ I don't care, $5=$ sure I am discipline $)$

6- Do you think COVID-19 pandemic has positive consequences?

$(1=$ Plenty, $5=$ Adverse effects $)$

7- I am afraid of being infected

$(1=$ Never , I don't care, $5=$ So afraid $)$

8- I am worry about my family.

$(1=$ Never, $5=$ Very worried $)$

9- I feel that COVID-19 is a punishment from Allah

$(1=I$ do not think so, $5=$ Surely $)$

10- I am hiding my fears about COVID-19 in front of others

$(1=$ Never, $5=$ yes, totally $)$

11- I feel helpless against COVID-19 pandemic

$(1=$ Never, $5=$ Completely $)$

12- I feel bored with COVID-19 quarantine

$(1=$ No, it is ok, $5=$ Extremely $)$

13- Quarantine is less harmful than infection

$(1=$ Surely - yes, $5=$ Quarantine is so boring $)$

14- I avoid dinning out/ordering food

$(1=$ Never, $5=$ Completely $)$

15- I avoid travelling/using public transport

$(1=$ Never, $5=$ Completely $)$

16- I clean surfaces daily

$(1=$ Never, $5=$ Completely $)$

17 - I am airing out my house

$(1=$ Never, $5=$ Completely $)$

18 - I wear a respirator upon leaving my house

$(1=$ Never, $5=$ All the time $)$

19- I wash my hands, especially when I get back home

(Never $=1$, Regularly $=5$ )

20 - I am scrubbing my hands for ... seconds

$(5 s=1,20 s=5)$

21- I wash my mouth with water and salt/vinegar

(Never $=1$, Always $=5)$

22- I take antibiotics and vitamins to protect myself from the infection

(Not at all $=1$, Certainly $=5$ )

23- I drink hot drinks to protect myself from the infection

(Never $=1$, All the time $=5$ )

24- Do you have adequate information about COVID-19 and its spread? (never $=1$, completely $=5$ )

25 - Does information you gathered on COVID-19 confuse you?

(Not at all $=1$, Totally confusing $=5$ )

Anxiety Disorder based on DSM-IV diagnostic criteria (American Psychiatric Association, 2000). The GAD-7 scale is utilized to assess symptom severity of anxiety. The Arabic Version of GAD-7 showed adequate psychometric properties in terms of reliability and structural validity (AlHadi et al., 2017; Sawaya et al., 2016).

\section{Statistical Analysis}

For estimating the internal consistency of CPRQ, we used Cronbach's Alpha $(\alpha)$, McDonald's Omega $(\omega)$, Inter-Item Correlations Mean (MII), and Item-Total Correlations Mean (MIT). Before deciding to extract CPRQ's factors, we investigated the suitable number of factors that fit the current data. We used both "Parallel Analysis" and "Very Simple Structure" of R, Psych Package (see http://personalityproject.org/r/psych/). Consequently, we conducted EFA for categorical data with a weighted least square mean and variance adjusted (WLSMV) and CF-Equamax rotation method using Mplus Program (Muthen \& Muthen, 2012). Using SPSS version 18, we utilized independent sample T-Test and ANOVA, to estimate the significant differences of means.

\section{Results}

\section{EFA: Number of Factors, Extraction, Final Model Structure, and Naming of Factors}

To identify a suitable number of factors that could fit the current data well, we used Parallel Analysis and Very Simple Structure tests. According to results from the above tests (See Figs. 1 and 2, and Supplementary Table 1), we estimated the significance of 5 to 8 -factor models. To choose the significant model from such factors, we considered the following 3 statistical assumptions: 1) good values of fit indices, 2) the existence of $\geq 3$ significant loaded variables on every single factor (Yong \& Pearce, 2013), 3) considerable loadings must be $>0.30$ (Yong \& Pearce, 2013). Depending upon the above considerations, we choose the 5 -factor model (See Table 3 and Supplementary Tables 2-4). The extraction of 5-factor model using WLSMV estimator and CF-Equamax rotation showed fair fit indices as CFI $=0.965$, TLI $=0.943$, RMSEA $=0.045$ (90\% C.I. ranged between 0.044 and 0.047), and SRMR $=0.025$. As results showed that items 6, 20, and 24 had no considerable loadings according to assumptions mentioned above (See Table 3), we considered ignoring such items in future analysis and discussion. However, we named factor 1 as Anxiousness, factor 2 as Social Support, factor 3 as Avoiding/Cleaning, factor 4 as Depressivity, and factor 5 as Medical Concern.

The first factor (Anxiousness) reflected the response of fear toward such a crisis. This factor describes risk perception, COVID-19's seriousness, risk of infection, personal concerns, and family-related worries. The second factor (Social Support) has reflected a behavioral response regarding the potential ability to avoid infection and how individuals support each other to face such pandemic. The third factor (Avoiding/Cleaning) has reflected a behavioral response demonstrated in the procedures that individuals follow to remedy 
Fig. 1 Number of factors using the Parallel Analysis

\section{Parallel Analysis Scree Plots}

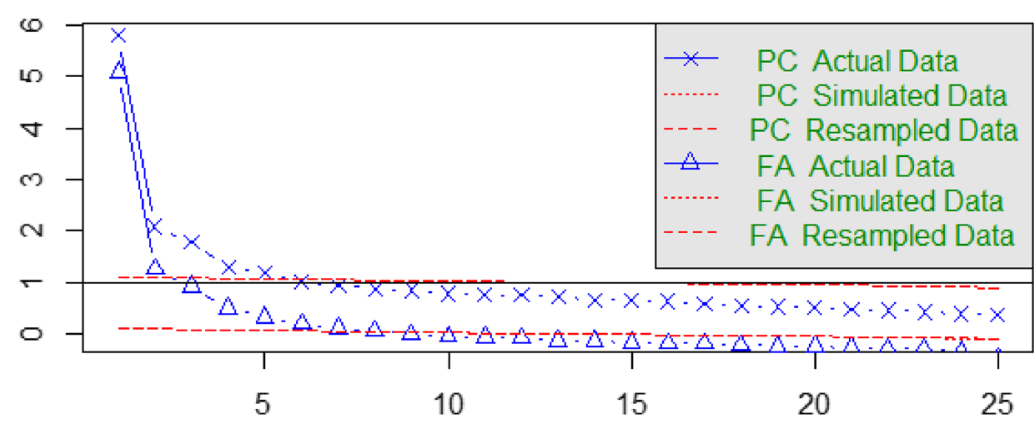

Factor/Component Number such a crisis. The fourth factor (Depressivity) has reflected a cognitive response of helplessness. The fifth factor (Medical Concern) has reflected a behavioral response of the bodyconcern procedures to avoid the infection.

\section{Internal Consistency}

As shown in Table 4, factor 4 (Depressivity) showed the lowest values across all the internal consistency tests. Further, factor 3 (Avoiding/Cleaning) showed the highest values in both of Alpha's and Omega's coefficients. Both second factor (Social Support) and fifth factor (Medical Concern) showed the highest values regarding the mean of item-total correlations. Also, the fifth factor showed the highest value regarding the mean of inter-item correlations. The total score of CPRQ showed good internal consistency. Moreover, both GAD-7 and PHQ-9 showed high values across all the tests of internal consistency with slightly higher values of PHQ-9. The results of Alpha, Omega, MII, and MIT showed fair values except for Depressivity. As the method used to select the sample included no prior investigation of psychopathology, we interpret such low internal consistency due to the heterogeneity nature of the sample. We meant by the heterogeneity that the participants who came to this study might differ in terms of psychopathology and normality.

\section{Correlations Matrix among Study Variables}

As Table 5 shows, both GAD-7 and PHQ-9 showed a significant moderate positive correlation with Depressivity and a significant low positive correlation with Anxiousness. Further, both GAD-7 and PHQ-9 had no significant correlations with Avoiding/Cleaning and Medical Concern. Besides, GAD-7 and PHQ-9 showed low negative significant values with Social Support.

\section{Differences between Subgroups}

As shown in Table 6, T-test results significantly differed between males and females as for Anxiuosness, Avoiding/ Cleaning, Depressivity, GAD-7, and PHQ-9. Regarding scores means, females had higher values in all of the above variables. Significant differences between rural and urban areas appeared in Social Support, Depressivity, Medical
Fig. 2 Number of factors using the Very Simple Structure analysis

\section{Very Simple Structure}

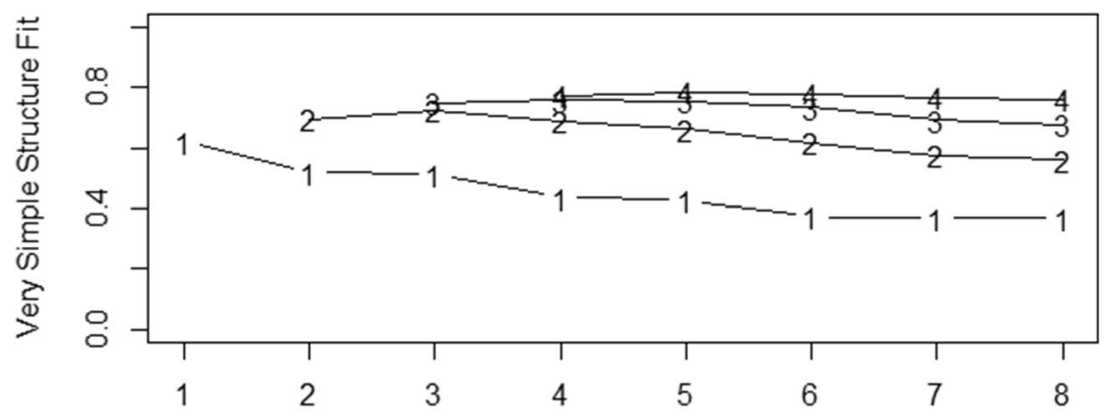

Number of Factors 
Table 3 The 5-Factor Structure of the CPRQ $(n=7.015)$

\begin{tabular}{|c|c|c|c|c|c|}
\hline Items & 1 & 2 & 3 & 4 & 5 \\
\hline How dangerous is COVID-19 to human life? & $0.715^{*}$ & $0.114^{*}$ & $-0.086^{*}$ & $0.097 *$ & -0.002 \\
\hline Risk of infection is & $0.640 *$ & $0.086^{*}$ & 0.015 & 0.014 & $-0.077^{*}$ \\
\hline Can you avoid infection? & $-0.110 *$ & $0.618^{*}$ & -0.015 & $0.052 *$ & $0.055^{*}$ \\
\hline Does your family help in avoiding infection? & $0.115^{*}$ & $0.795 *$ & $-0.056^{*}$ & $0.032 *$ & $0.029 *$ \\
\hline Do you help your family to avoid infection? & $0.164 *$ & $0.687 *$ & $0.135 *$ & 0.000 & $0.061^{*}$ \\
\hline Do you think COVID-19 pandemic has positive consequences? & $-0.263 *$ & $0.219^{*}$ & $0.070^{*}$ & 0.029 & $0.042 *$ \\
\hline I am afraid of being infected & $0.512 *$ & $-0.091 *$ & $0.033^{*}$ & $0.243 *$ & $0.213^{*}$ \\
\hline I am worry about my family & $0.430 *$ & $0.134 *$ & $0.202 *$ & $0.325 *$ & $-0.132 *$ \\
\hline I feel that COVID-19 is a punishment from Allah & 0.025 & $0.143^{*}$ & $-0.119 *$ & $0.465^{*}$ & $0.111^{*}$ \\
\hline I am hiding my fears about COVID-19 in front of others & $-0.288 *$ & $0.243 *$ & 0.022 & $0.401 *$ & -0.020 \\
\hline I feel helpless against COVID-19 pandemic & $0.104 *$ & $-0.162 *$ & $0.052 *$ & $0.596 *$ & -0.003 \\
\hline I feel bored with COVID-19 quarantine & 0.012 & $0.034^{*}$ & $-0.077 *$ & $0.480 *$ & -0.015 \\
\hline Quarantine is less harmful than infection & $0.246^{*}$ & $0.101 *$ & $0.511 *$ & $0.137 *$ & $-0.165^{*}$ \\
\hline I avoid dinning out/ordering food & $0.042 *$ & -0.002 & $0.670^{*}$ & $0.117 *$ & 0.011 \\
\hline I avoid travelling/using public transport & $0.045^{*}$ & 0.009 & $0.687 *$ & $0.119 *$ & -0.012 \\
\hline I clean surfaces daily & $0.116^{*}$ & $0.252^{*}$ & $0.444 *$ & $0.029 *$ & $0.275^{*}$ \\
\hline I am airing out my house & $0.092 *$ & $0.283^{*}$ & $0.508 *$ & $0.099 *$ & $0.081 *$ \\
\hline I wear a respirator upon leaving my house & $0.063^{*}$ & $0.166^{*}$ & $0.380 *$ & $-0.092 *$ & $0.363-$ \\
\hline I wash my hands, especially when I get back home & $0.153 *$ & $0.226^{*}$ & $0.577 *$ & 0.005 & $0.046^{*}$ \\
\hline I am scrubbing my hands for ... seconds & $0.098^{*}$ & $0.051^{*}$ & $0.247 *$ & $-0.194 *$ & $0.108^{*}$ \\
\hline I wash my mouth with water and salt/vinegar & $-0.037 *$ & $0.064 *$ & -0.023 & -0.010 & $0.780 *$ \\
\hline I take antibiotics and vitamins to protect myself from the infection & -0.011 & $-0.020^{*}$ & $-0.068 *$ & $0.067 *$ & $0.845^{*}$ \\
\hline I drink hot drinks to protect myself from the infection & $0.075 *$ & $0.099 *$ & $0.136^{*}$ & $0.060 *$ & $0.591 *$ \\
\hline Do you have adequate information about COVID-19 and its spread? & $0.089^{*}$ & $0.219 *$ & $0.116^{*}$ & $0.050 *$ & $0.175^{*}$ \\
\hline Does information you gathered on COVID-19 make you confused? & $0.107 *$ & $-0.085^{*}$ & $0.038^{*}$ & $0.431 *$ & $0.181 *$ \\
\hline
\end{tabular}

Note: loadings $\geq 0.30$ are in bold

Concern, and PHQ-9. The urban group had higher values in the above factors except for the Medical Concern. Further, ANOVA results showed significant differences among age groups in all study variables. The 18-21-year-old group had higher values amongst other age groups concerning Anxiousness, Depressivity, GAD-7, and PHQ-9. The 41-50 and 51-60-year-old groups showed higher values for Social Support, and the 41-50-year-old group showed a higher value in Avoiding/Cleaning. Moreover, the 51-60-year-old group showed a higher value in Medical Concern. Among the socioeconomic groups, results revealed significant differences across all study variables. The "Very High Level" group
Table 4 Descriptive and Reliability of Study Variables

\begin{tabular}{lrrrrrrrr}
\hline Variables $(n=7015)$ & M & SD & Skew & Kurt & MIT & MII & $\alpha$ & $\omega$ \\
\hline F1: Anxiousness (4-item) & 4.24 & 0.71 & -1.22 & 1.80 & 0.47 & 0.37 & 0.70 & 0.75 \\
F2: Social Support (3-item) & 3.91 & 0.85 & -0.67 & 0.13 & 0.54 & 0.46 & 0.72 & 0.74 \\
F3: Avoiding and Cleaning (7-item) & 4.10 & 0.78 & -0.99 & 0.71 & 0.44 & 0.37 & 0.81 & 0.86 \\
F4: Depressivity (5-item) & 3.50 & 0.78 & -0.24 & -0.16 & 0.29 & 0.18 & 0.53 & 0.57 \\
F5: Medical Concern (3-item) & 2.50 & 1.12 & 0.49 & -0.57 & 0.54 & 0.47 & 0.73 & 0.74 \\
CPRQ (22-item) & 3.74 & 0.57 & -0.62 & 1.20 & 0.40 & 0.20 & 0.85 & 0.87 \\
GAD-7 & 20.20 & 7.17 & 0.06 & -0.73 & 0.62 & 0.47 & 0.86 & 0.89 \\
PHQ-9 & 24.84 & 9.64 & 0.20 & -0.83 & 0.68 & 0.52 & 0.91 & 0.92 \\
\hline
\end{tabular}

Note. $\mathrm{M}=$ Mean, $\mathrm{SD}=$ Standard Deviation, Skew $=$ Skewness, Kurt $=$ Kurtosis, MIT $=$ Mean Item Total correlations, $\mathrm{MII}=$ Mean Inter-Item correlations, $\alpha=$ Cronbach's Alpha, and $\omega=$ McDonald's Omega 
Table 5 Correlations Matrix of Study Variables $(\mathrm{n}=7015)$

\begin{tabular}{lllllll}
\hline Variables & Anxiousness & $\begin{array}{l}\text { Social } \\
\text { Support }\end{array}$ & $\begin{array}{l}\text { Avoiding and } \\
\text { Cleaning }\end{array}$ & Depressivity & $\begin{array}{l}\text { Medical } \\
\text { Concern }\end{array}$ & $\begin{array}{l}\text { GAD- } \\
7\end{array}$ \\
\hline Social Support & $\mathbf{. 3 5 0}$ & & & & \\
Avoiding and Cleaning & $\mathbf{. 4 8 3}$ & $\mathbf{. 5 2 8}$ & & & \\
Depressivity & $\mathbf{. 3 5 7}$ & $\mathbf{. 1 9 3}$ & $\mathbf{. 2 5 5}$ & $\mathbf{. 2 1 4}$ & \\
Medical Concern & $\mathbf{. 1 6 5}$ & $\mathbf{. 3 0 6}$ & $\mathbf{. 3 3 5}$ & $\mathbf{. 3 3 5}$ & .003 \\
GAD-7 & $\mathbf{. 1 7 9}$ & $\mathbf{- . 0 6 1}$ & .002 & $\mathbf{. 3 3 5}$ \\
PHQ-9 & $\mathbf{. 1 4 3}$ & $\mathbf{- . 0 6 7}$ & -.022 & $\mathbf{. 3 0 4}$ & .002 & $\mathbf{. 8 4 0}$ \\
\hline
\end{tabular}

Note: all significant values at 0.01 are in bold showed higher values across all study variables except GAD-7 and PHQ-9. The "Moderate" group showed the highest value in GAD-7, and the "Low" group showed the highest value in PHQ-9. Moreover, there were significant differences among the educational groups across all study variables. However, the results showed that the "Ph.D." group had the highest values among all study variables except Depressivity, GAD7, and PHQ-9. Both of "Read and Write" and "High School" groups had the highest values in Depressivity. Besides, the "High School" group had the highest values in GAD-7 and PHQ-9.

\section{Discussion}

\section{The Current Model}

To answer the first question of the study "how participants psychologically response to the COVID-19 outbreak?", we constructed a new questionnaire that revealed a 5-factor model. Such a 5-factor model describes participants' psychological responses to the COVID-19 outbreak. Our findings on the first factor (Anxiousness) as a response to COVID-19 found to be consistent with several studies that have reported the related anxiety, fear, and worry with COVID-19 outbreak (Ahmed et al., 2020; Ahorsu et al., 2020; Asmundson et al., 2020; Cao et al., 2020; Huang \& Zhao, 2020; Lee, 2020; Özdin \& Bayrak Özdin, 2020; Wang et al., 2020). Our findings for Social Support found to be consistent with previous studies that have indicated that social support is a psychosocial response during such a crisis (El-Zoghby et al., 2020; Zhang \& Ma, 2020). The findings of Harper et al. (2020) supports our findings regarding Avoiding/Cleaning as a response to COVID-19. They have reported incremental behaviors to avoid contamination (e.g., social distancing, improved hand hygiene) to COVID-19. Our conclusions regarding
Depressivity were found to be consistent with several studies that have discussed the helplessness and hopelessness as a response to COVID-19 outbreak (Shaw, 2020; Szabo et al., 2020; Montemurro, 2020). Finally, the fifth factor (Medical Concern) results are consistent with the findings of Harper et al. (2020), indicating that preventive behaviors increased during this crisis.

\section{The Current Model and Previous Relevant Models}

By comparing our study findings with other relevant models and theories, we found that the first factor (Anxiousness) reflects the beliefs of susceptibility, severity, and fear regarding the protection motivation theory (Rogers, 1975, 1983). In addition, we found the second factor (Social Support) resembles the concept of health locus of control of the attribution theory (Kelley, 1967) and both of response effectiveness and selfefficacy beliefs of the protection motivation theory (Rogers, 1975, 1983), which describes individuals' beliefs of their ability to avoid infection. Further, we found that both of the third and fifth factors (Avoiding and Cleaning, and Medical Concern) reflect the coping behaviors as indicated by Leventhal et al. (1980). Finally, the fourth factor (Depressivity) reflects unhealthy (maladjustment) behaviors of the crisis theory (Moos \& Schaefer, 1984), in which individuals respond to the pandemic with helplessness beliefs.

\section{The Current Model and Psychopathology}

Regarding the second question of this study, we estimated a correlation matrix among the study variables. The positive significant correlation between Anxiousness and both Generalized Anxiety Disorder (GAD; as measured by GAD7) and Major Depressive Disorder (MDD; as measured by PHQ-9), further supports the validity of Anxiousness factor. However, a low value of such correlations confirmed that 


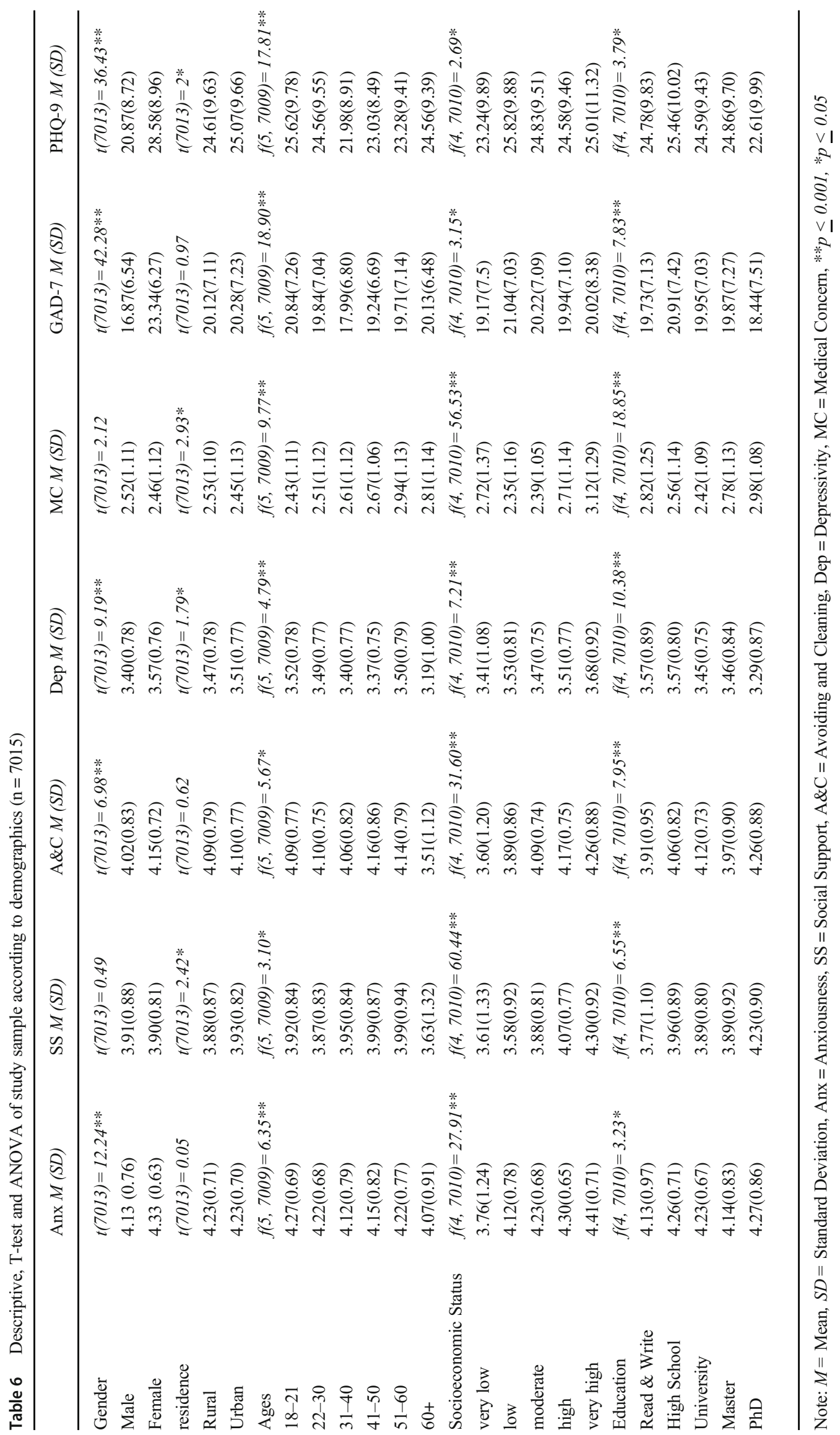


Anxiousness does not relate to psychopathology but rather is considered as a response to the COVID-19 outbreak. The correlation between Social Support and both GAD and MDD revealed a significant negative value. This finding also confirms the validity of Social Support. The significant positive correlations between Depressivity and both GAD and MDD confirm that this factor is considered as a maladaptive response to COVID-19. However, the moderate value of such correlations keeps Depressivity factor away from psychopathology.

\section{The Current Model, Psychopathology, and Demographics}

To address the third question of this study, we explored differences among study subgroups to identify such differences across the study variables. The findings referred that females have higher levels of Anxiousness, Avoiding/Cleaning, Depressivity, GAD, and MDD than males. Further, urban areas have higher levels of Social Support, Depressivity, GAD, and MDD than rural areas. The 18-21-year-old group showed the highest level of Anxiousness, Depressivity, GAD, and MDD as compared to other age groups. Such findings were found to be consistent with previous studies (Huang et al., 2020; Qiu et al., 2020). Further, there has been a partial inconsistency with another study, which reported that living in urban areas is considered a protective factor against anxiety during such a crisis (Cao et al., 2020).

We interpret the higher values of females as they tend to be more vulnerable to emotional stress than males. Accordingly, females may take more precautions to relieve such emotions. However, other females may experience more stress if they adopted negative coping strategies. Regarding the place of residence, urban areas are suffering from many stressors in general (e.g., crowding and time constraints), and such stressors may increase the psychological stress and trigger coping strategies whatever positive or negative. In terms of ages, participants aged between 18 and 21 have many stressors (e.g., study burden and emotional problems related to such developmental level). Such stressors may lead to further psychological stress during such a crisis. Due to the nature of such a developmental level, the quarantine procedures may trigger more stressful feelings.

Moreover, the higher educated participants (Ph.D. group) showed higher levels for Anxiousness, Social Support, Avoiding/Cleaning, and Medical Concern. Such findings revealed higher levels of risk perception and positive practices against COVID-19. Accordingly, the higher educated participants have the potential to practice more positively as compared to the lower educated people against the COVID-19 outbreak. However, such results were found to be inconsistent with the study Qiu et al. (2020), as they have reported that the higher educated participants have a higher tendency to stress.
Furthermore, the group with a very high level of socioeconomic status (SES) showed high levels across all study variables. Further, the group with a moderate level of SES showed a high level of GAD, and the group with a low level of SES showed a high level of MDD. Such findings are partially consisted of Cao et al. (2020), as they reported that income stability is a psychological protective factor against COVID-19 pandemic. We suppose that the participants with a higher level of SES have more ability to gather information about COVID-19 without investigating the validity thereof. Under such circumstances, the level of psychological stress may increase. As for the high level of SES, the participants tend to have more work and entertainment activities. Therefore, such quarantine procedures may lead to further psychological stress.

This study findings provide a holistic model that explains the psychological response toward the COVID-19 pandemic. This model reflects most of the relevant health-related models and theories, which describe healthy and unhealthy responses. In this study, healthy behaviors included risk perception (normal fear), social support, and protective measures. Unhealthy behaviors (maladjustment behaviors) were represented in feeling hopeless and helplessness. Further, we linked our model to psychopathology (depression and anxiety). Further, the findings explained the psychological response and psychopathology in terms of demographic characteristics during the crisis. Finally, this study improves our understanding of the psychological response toward the pandemic in a broader sense not only from a psychopathology perspective.

In this study, psychopathological problems appeared more frequently in females, urban areas, 18- to 21-yearolds, poorly educated, and low SES groups than other groups. Accordingly, we consider such segments need more attention regarding psychological intervention. Further, psychological well-being of those who suffer stressors should be considered during COVID-19 pandemic. Finally, resources should be provided to promote positive practices (e.g. physical excersices, healthy food, sleep hygiene, avoidance of excessive alcohol, tobacco, substance abuse, etc.).

\section{Strength and Limitations}

The strength of this study lies in developing a new factorial model for psychological response to the COVID-19 pandemic. Further, such a model is derived directly from participants' thoughts during such a crisis. The limitation of this study is that it has not included psychopathological samples. 


\section{Future Research}

For further research regarding our findings, we suggest exploring the current model cross-culturally to discuss generalizability. Further, we recommend investigating the potential risk factors (e.g., psychopathology, poor income and low education) that may affect mental health during COVID-19 pandemic.

\section{Conclusion}

In general, the COVID-19 pandemic has a negative impact on public mental health and psychological well-being. In this study, the findings revealed that COVID-19 pandemic triggered specific responses. Such responses include normal fear due to risk perception, positive practices (Social support and protective measures), negative coping strategies, and drug consumption (antibiotics and supplements). Further, the study showed a correlation between negative coping responses and both major depressive disorder and generalized anxiety disorder. Moreover, females, urban areas, university students, and aged participants were more prone to psychopathology. Further, poorly educated, and low SES groups were more likely to develop psychopathological symptoms. Finally, this is the first study that timely provides a structured model exploring the psychological response towards COVID-19 on an Egyptian community sample.

Supplementary Information The online version contains supplementary material available at https://doi.org/10.1007/s12144-021-01701-y.

Data Availability The raw data available upon request.

\section{Declarations}

All procedures performed in studies involving human participants were following the ethical standards of the institutional research committee (Kafrelshiekh university, ethical code: not applicable) and with the 1964 Helsinki Declaration and later amendments or comparable ethical standards thereof.

Informed Consent (when Applicable) Informed consent was obtained from all individual adult participants included in the study.

Conflicting Interests The author(s) declared no potential conflicts of interest concerning the research, authorship, and/or publication of this article.

\section{References}

Abdel Wahed, W. Y., Hefzy, E. M., Ahmed, M. I., \& Hamed, N. S. (2020). Assessment of knowledge, attitudes, and perception of health care workers regarding COVID-19, a cross-sectional study from Egypt. Journal of Community Health, 45(6), 1242-1251. https://doi.org/10.1007/s10900-020-00882-0.

Abdelghani, M., El-Gohary, H. M., Fouad, E., \& Hassan, M. S. (2020). Addressing the relationship between perceived fear of COVID-19 virus infection and emergence of burnout symptoms in a sample of Egyptian physicians during COVID-19 pandemic: A cross-sectional study. Middle East Current Psychiatry, 27(1). https://doi.org/10. 1186/s43045-020-00079-0.

Abdelhafiz, A. S., Ali, A., Ziady, H. H., Maaly, A. M., Alorabi, M., \& Sultan, E. A. (2020a). Prevalence, associated factors, and consequences of burnout among Egyptian physicians during COVID-19 pandemic. Frontiers in Public Health, 8. doi:https://doi.org/10. 3389/fpubh.2020.590190.

Abdelhafiz, A. S., Mohammed, Z., Ibrahim, M. E., Ziady, H. H., Alorabi, M., Ayyad, M., \& Sultan, E. A. (2020b). Knowledge, perceptions, and attitude of egyptians towards the novel coronavirus disease (COVID-19). Journal of Community Health, 45(5), 881-890. https://doi.org/10.1007/s10900-020-00827-7.

Ahmed, M. Z., Ahmed, O., Aibao, Z., Hanbin, S., Siyu, L., \& Ahmad, A. (2020). Epidemic of COVID-19 in China and associated psychological problems. Asian Journal of Psychiatry, 51, 102092. https://doi. org/10.1016/j.ajp.2020.102092.

Ahorsu, D. K., Lin, C., Imani, V., Saffari, M., Griffiths, M. D., \& Pakpour, A. H. (2020). The fear of COVID-19 scale: Development and initial validation. International Journal of Mental Health and Addiction. doi:https://doi.org/10.1007/s11469-02000270-8.

Ajzen, I. (1985). From intentions to actions: A theory of planned behavior. In action control (pp. 11-39). Springer, Berlin, Heidelberg.

Alamrawy, R. G., Fadl, N., \& Khaled, A. (2021). Psychiatric morbidity and dietary habits during COVID-19 pandemic: A cross-sectional study among Egyptian youth (14-24 years). Middle East Current Psychiatry, 28(1). https://doi.org/10.1186/s43045-021-00085-w.

AlHadi, A. N., AlAteeq, D. A., Al-Sharif, E., Bawazeer, H. M., Alanazi, H., AlShomrani, A. T., et al. (2017). An Arabic translation, reliability, and validation of patient health questionnaire in a Saudi sample. Annals of General Psychiatry, 16(1), 32. https://doi.org/10.1186/ s12991-017-0155-1.

American Psychiatric Association. (2000). Diagnostic and statistical manual of mental disorders, text revision (DSM-IV-TR) (4th ed.). Author.

Arafa, A., Mohamed, A., Saleh, L., \& Senosy, S. (2020). Psychological impacts of the COVID-19 pandemic on the public in Egypt. Community Mental Health Journal, 57(1), 64-69. https://doi.org/ 10.1007/s10597-020-00701-9.

Arafa, A., Mohammed, Z., Mahmoud, O., Elshazley, M., \& Ewis, A. (2021). Depressed, anxious, and stressed: What have healthcare workers on the frontlines in Egypt and Saudi Arabia experienced during the COVID-19 pandemic? Journal of Affective Disorders, 278, 365-371. https://doi.org/10.1016/j.jad.2020.09.080.

Asmundson, G. J., Paluszek, M. M., Landry, C. A., Rachor, G. S., McKay, D., \& Taylor, S. (2020). Do pre-existing anxiety-related and mood disorders differentially impact COVID-19 stress responses and coping? Journal of Anxiety Disorders, 74, 102271. https://doi.org/10.1016/j.janxdis.2020.102271.

Bao, Y., Sun, Y., Meng, S., Shi, J., \& Lu, L. (2020). 2019-nCoV epidemic: Address mental health care to empower society. The Lancet, 395(10224), e37-e38. https://doi.org/10.1016/s0140-6736(20) 30309-3.

Becker, M. H., Maiman, L. A., Kirscht, J. P., Haefner, D. P., \& Drachman, R. H. (1977). The health belief model and prediction of dietary compliance: A field experiment. Journal of Health and Social Behavior, 18, 348-366.

Cao, W., Fang, Z., Hou, G., Han, M., Xu, X., Dong, J., \& Zheng, J. (2020). The psychological impact of the COVID-19 epidemic on 
college students in China. Psychiatry Research, 287, 112934. https://doi.org/10.1016/j.psychres.2020.112934.

Elkholy, H., Tawfik, F., Ibrahim, I., Salah El-din, W., Sabry, M., Mohammed, S., ... Omar, A. N. (2020). Mental health of frontline healthcare workers exposed to COVID-19 in Egypt: A call for action. International Journal of Social Psychiatry, 002076402096019. doi:https://doi.org/10.1177/0020764020960192.

El-Zoghby, S. M., Soltan, E. M., \& Salama, H. M. (2020). Impact of the COVID-19 pandemic on mental health and social support among adult egyptians. Journal of Community Health, 45(4), 689-695. https://doi.org/10.1007/s10900-020-00853-5.

Engel, G. L. (1977). The need for a new medical model: A challenge for biomedicine. Science, 196(4286), 129-136.

Gao, J., Zheng, P., Jia, Y., Chen, H., Mao, Y., Chen, S., ... Wang, Y. (2020). Mental health problems and social media exposure during COVID-19 outbreak. SSRN Electronic Journal. doi:https://doi.org/ $10.2139 /$ ssrn.3541120.

Ghazawy, E. R., Ewis, A. A., Mahfouz, E. M., Khalil, D. M., Arafa, A., Mohammed, Z., Mohammed, E. N. F., Hassan, E. E., Abdel Hamid, S., Ewis, S. A., \& Mohammed, A. E. N. S. (2020). Psychological impacts of COVID-19 pandemic on the university students in Egypt. Health Promotion International.

Harper, C. A., Satchell, L. P., Fido, D., \& Latzman, R. D. (2020). Functional fear predicts public health compliance in the COVID19 pandemic. International Journal of Mental Health and Addiction. https://doi.org/10.1007/s11469-020-00281-5.

Huang, L., Xu, F. M., \& Liu, H. R. (2020). Emotional responses and coping strategies of nurses and nursing college students during COVID-19 outbreak. doi:https://doi.org/10.1101/2020.03.05. 20031898.

Huang, Y., \& Zhao, N. (2020). Generalized anxiety disorder, depressive symptoms and sleep quality during COVID-19 epidemic in China: A web-based cross-sectional survey. Doi:https://doi.org/10.21203/ rs.3.rs- $17172 / \mathrm{v} 1$.

Kelley, H. H. (1967). Attribution theory in social psychology. In Nebraska symposium on motivation. University of Nebraska Press.

Khalaf, O. O., Khalil, M. A., \& Abdelmaksoud, R. (2020). Coping with depression and anxiety in Egyptian physicians during COVID-19 pandemic. Doi:https://doi.org/10.21203/rs.3.rs-84013/v1.

Kwok, K. O., Li, K. K., Chan, H. H., Yi, Y. Y., Tang, A., Wei, W. I., \& Wong, Y. S. (2020). Community responses during the early phase of the COVID-19 epidemic in Hong Kong: Risk perception, information exposure and preventive measures. doi:https://doi.org/10.1101/ 2020.02.26.20028217.

Lazzerini, M., \& Putoto, G. (2020). COVID-19 in Italy: Momentous decisions and many uncertainties. The Lancet Global Health, 8(5), e641-e642. https://doi.org/10.1016/s2214-109x(20)30110-8.

Lee, S. A. (2020). Coronavirus anxiety scale: A brief mental health screener for COVID-19 related anxiety. Death Studies, 44(7), 393-401. https://doi.org/10.1080/07481187.2020.1748481.

Leventhal, H., Meyer, D., \& Nerenz, D. (1980). The common sense representation of illness danger. Contributions to medical psychology, 2, 7-30.

Liu, N., Zhang, F., Wei, C., Jia, Y., Shang, Z., Sun, L., et al. (2020). Prevalence and predictors of PTSS during COVID-19 outbreak in China hardest-hit areas: Gender differences matter. Psychiatry Research, 287, 112921. https://doi.org/10.1016/j.psychres.2020. 112921.

Montemurro, N. (2020). The emotional impact of COVID-19: From medical staff to common people. Brain, Behavior, and Immunity, 87, 23-24. https://doi.org/10.1016/j.bbi.2020.03.032.

Moos, R. H., \& Schaefer, J. A. (1984). The crisis of physical illness. In coping with physical illness (pp. 3-25). Springer, Boston, MA.

Muthen, L. K., \& Muthen, B. (2012). Mplus: Statistical analysis with latent variables: user's guide. Muthén \& Muthén.
Ogden, J. (2004). Health psychology: A textbook (3rd ed.). England: Open University Press.

Özdin, S., \& Bayrak Özdin, Ș. (2020). Levels and predictors of anxiety, depression and health anxiety during COVID-19 pandemic in Turkish society: The importance of gender. International Journal of Social Psychiatry, 66(5), 504-511. https://doi.org/10.1177/ 0020764020927051.

Pennycook, G., McPhetres, J., Zhang, Y., \& Rand, D. G. (2020). Fighting COVID-19 misinformation on social media: Experimental evidence for a scalable accuracy nudge intervention. Doi:https://doi.org/10. 31234/osf.io/uhbk9.

Prochaska, J. O., \& DiClemente, C. C. (1982). Transtheoretical therapy: Toward a more integrative model of change. Psychotherapy: Theory, research \& practice, 19(3), 276.

Qian, M., Wu, Q., Wu, P., Hou, Z., Liang, Y., Cowling, B. J., \& Yu, H. (2020). Psychological responses, behavioral changes and public perceptions during the early phase of the COVID-19 outbreak in China: A population based cross-sectional survey. doi:https://doi.org/10. 1101/2020.02.18.20024448.

Qiu, J., Shen, B., Zhao, M., Wang, Z., Xie, B., \& Xu, Y. (2020). A nationwide survey of psychological distress among Chinese people in the COVID-19 epidemic: Implications and policy recommendations. General Psychiatry, 33(2), e100213. https://doi.org/10.1136/ gpsych-2020-100213.

Rogers, R. W. (1975). A protection motivation theory of fear appeals and attitude change1. The Journal of Psychology, 91(1), 93-114.

Rogers, R. W. (1983). Cognitive and psychological processes in fear appeals and attitude change: A revised theory of protection motivation. Social psychophysiology: A sourcebook, 153-176.

Said, R. M., \& El-Shafei, D. A. (2020). Occupational stress, job satisfaction, and intent to leave: Nurses working on front lines during COVID-19 pandemic in Zagazig city, Egypt. Environmental Science and Pollution Research, 28(7), 8791-8801. https://doi.org/ 10.1007/s11356-020-11235-8.

Saleh, M. S. (2020). The "stay at home" orders effect on mental health of Egyptian adults during the COVID-19 pandemic partial lockdown. International Journal of Human Rights in Healthcare, ahead-ofprint (ahead-of-print). doi:https://doi.org/10.1108/ijhrh-08-20200065.

Sawaya, H., Atoui, M., Hamadeh, A., Zeinoun, P., \& Nahas, Z. (2016). Adaptation and initial validation of the patient health questionnaire9 (PHQ-9) and the generalized anxiety disorder -7 questionnaire (GAD-7) in an Arabic speaking lebanese psychiatric outpatient sample. Psychiatry Research, 239, 245-252. https://doi.org/10.1016/j. psychres.2016.03.030.

Sehsah, R., Gaballah, M. H., El-Gilany, A., \& Albadry, A. A. (2021). Psychological distress among Egyptian physicians during COVID19 pandemic. International Archives of Occupational and Environmental Health. doi:https://doi.org/10.1007/s00420-02001624-4.

Shaw, S. C. (2020). Hopelessness, helplessness and resilience: The importance of safeguarding our trainees' mental wellbeing during the COVID-19 pandemic. Nurse Education in Practice, 44, 102780. https://doi.org/10.1016/j.nepr.2020.102780.

Szabo, T. G., Richling, S., Embry, D. D., Biglan, A., \& Wilson, K. G. (2020). From helpless to hero: Promoting values-based behavior and positive family interaction in the midst of COVID-19. Behavior Analysis in Practice., 13, 568-576. https://doi.org/10.1007/ s40617-020-00431-0.

Wang, C., Pan, R., Wan, X., Tan, Y., Xu, L., Ho, C. S., \& Ho, R. C. (2020). Immediate psychological responses and associated factors during the initial stage of the 2019 coronavirus disease (COVID-19) epidemic among the general population in China. International Journal of Environmental Research and Public Health, 17(5), 1729. https://doi.org/10.3390/ijerph17051729. 
Weinstein, N. D. (1983). Reducing unrealistic optimism about illness susceptibility. Health Psychology, 2(1), 11-20.

Weinstein, N. D. (1984). Why it won't happen to me: Perceptions of risk factors and susceptibility. Health Psychology, 3(5), 431-457.

Yang, Y., Li, W., Zhang, Q., Zhang, L., Cheung, T., \& Xiang, Y. (2020). Mental health services for older adults in China during the COVID19 outbreak. The Lancet Psychiatry, 7(4), e19. https://doi.org/10. 1016/s2215-0366(20)30079-1.

Yao, H., Chen, J., \& Xu, Y. (2020). Rethinking online mental health services in China during the COVID-19 epidemic. Asian Journal of Psychiatry, 50, 102015. https://doi.org/10.1016/j.ajp.2020. 102015.

Yong, A. G., \& Pearce, S. (2013). A beginner's guide to factor analysis: Focusing on exploratory factor analysis. Tutorials in quantitative methods for psychology, 9(2), 79-94.

Youssef, N., Mostafa, A., Ezzat, R., Yosef, M., \& El Kassas, M. (2020). Mental health status of health-care professionals working in quarantine and non-quarantine Egyptian hospitals during the COVID-19 pandemic. Eastern Mediterranean Health Journal, 26(10), 11551164. https://doi.org/10.26719/emhj.20.116.

Zhang, J., Wu, W., Zhao, X., \& Zhang, W. (2020). Recommended psychological crisis intervention response to the 2019 novel coronavirus pneumonia outbreak in China: A model of West China hospital. Precision Clinical Medicine, 3(1), 3-8. https://doi. org/10.1093/pcmedi/pbaa006.

Zhang, Y., \& Ma, Z. F. (2020). Impact of the COVID-19 pandemic on mental health and quality of life among local residents in Liaoning province, China: A cross-sectional study. International Journal of Environmental Research and Public Health, 17(7), 2381. https:// doi.org/10.3390/ijerph17072381.

Zhong, B., Luo, W., Li, H., Zhang, Q., Liu, X., Li, W., \& Li, Y. (2020). Knowledge, attitudes, and practices towards COVID-19 among Chinese residents during the rapid rise period of the COVID-19 outbreak: A quick online cross-sectional survey. International Journal of Biological Sciences, 16(10), 1745-1752. https://doi.org/ 10.7150/ijbs.45221.

Zhou, X., Snoswell, C. L., Harding, L. E., Bambling, M., Edirippulige, S., Bai, X., \& Smith, A. C. (2020). The role of Telehealth in reducing the mental health burden from COVID-19. Telemedicine and $e$ Health, 26(4), 377-379. https://doi.org/10.1089/tmj.2020.0068.

Publisher's note Springer Nature remains neutral with regard to jurisdictional claims in published maps and institutional affiliations. 